\title{
EXCLUSIVE MESON PRODUCTION AT COMPASS
}

\author{
Josef Pochodzalla $^{a}$, Lech Mankiewicz ${ }^{b}$, Murray Moinester $^{c}$, Gunther Piller $^{b}$, \\ Andrzej Sandacz ${ }^{d}$, Marc Vanderhaeghen ${ }^{e}$ \\ ${ }^{a}$ Max-Planck-Institut für Kernphysik, 69029 Heidelberg, Germany \\ ${ }^{b}$ Physics Department, Technical University Munich, D-85747 Garching, Germany \\ ${ }^{c}$ School of Physics and Astronomy, R. and B. Sackler Faculty of Exact Sciences, \\ Tel Aviv University, 69978 Ramat Aviv, Israel \\ ${ }^{d}$ Soltan Institute for Nuclear Studies, 00681 Warsaw, Poland \\ ${ }^{e}$ University Mainz, D-55099 Mainz, Germany
}

\begin{abstract}
We explore the feasibility to study exclusive meson production (EMP) in hard muon-proton scattering $\mu p \rightarrow \mu p M$ at the COMPASS experiment. These measurements constrain the off-forward parton distributions (OFPD's) of the proton, which are related to the quark orbital contribution to the proton spin.
\end{abstract}

\section{Introduction}

The internal structure of the proton has been a subject of high interest for many decades. In general, the spin of the proton resides in its quark and gluon constituents and in their orbital angular momenta:

$$
\frac{1}{2}\left\langle\Delta q_{v}\right\rangle+\frac{1}{2}\langle\Delta S\rangle+\langle\Delta G\rangle+L_{q}+L_{g}=\frac{1}{2}
$$

Here, $\frac{1}{2}\left\langle\Delta q_{v}\right\rangle$ denotes the spin in the valance quarks, $\frac{1}{2}\langle\Delta S\rangle$ is the total spin carried by the sea quarks and antiquarks, $\langle\Delta G\rangle$ represents the spin residing in the gluonic field, $L_{q}$ and $L_{g}$ represent the orbital momenta of the quarks and gluons, respectively. A main goal of the COMPASS experiment at CERN is the determination of the gluon contribution $\Delta G$. In COMPASS, $\Delta G$ will be studied via the photon-gluon fusion process, tagged either by charm-anticharm production, or by high- $p_{T}$ hadron pair productionl.

A factorization theorem for deeph-virtual Compton scattering (DVCS) was provided by $\mathrm{Ji}^{2}$ and Radyushkin $\mathrm{H}^{\mathrm{A}}$. For exclusive meson production by longitudinally polarized photons, a factorization theorem was proven recently

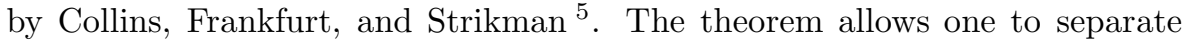
the meson production amplitudes into a perturbatively calculable component, describing the interaction of the virtual photon with quarks and gluons of the target, and a matrix element component which contains all information about the long-distance non-perturbative strong interaction dynamics in the produced meson and the nucleon target. The latter component can be parameterized, at leading order PQCD, in terms of 4 generalized structure functions, 


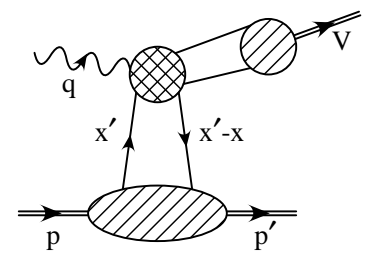

Figure 1: Illustration of the factorization theorem for exclusive meson production $\mathrm{E}$.

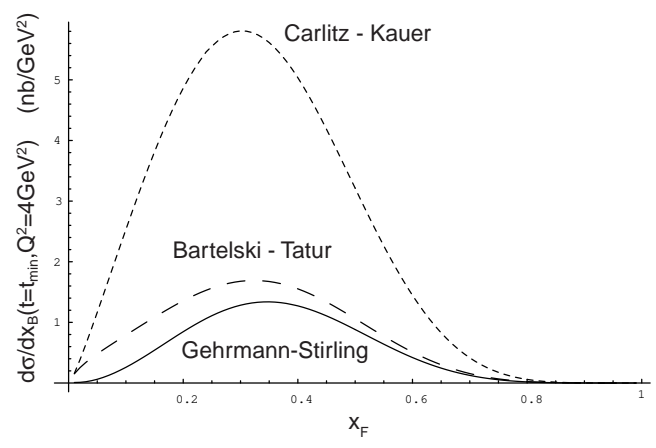

Figure 2: Differential cross section for $\pi^{0}$ production at $\mathrm{t}=\mathrm{t}_{\min }, Q^{2}=4 \mathrm{GeV}^{2}$, as a function of $\mathrm{x}_{B}$, in units of $n b / G e V^{2}$, for different valence quark parametrizations 15.16 .17 .18$.

the so called skewed or off-forward parton distributions (OFPD's). Ji showed 2 that the second moment of these OFPD's determines the total quark angular momentum, the sum of the intrinsic quark spins and their orbital angular momentum. The OFPD's differ from the usual parton distributions probed in inclusive reactions by having a non-zero momentum transfer between the proton in the initial and final state. Therefore, the OFPD's are not accessible in standard inclusive measurements. They can, however, be measured in deeply-virtual Compton scattering or in hard exclusive lepto-production of mesons (EMP). Thus, exclusive meson production provides a unique tool to explore the contribution of orbital angular momentum and the valance quark polarization to the nucleon spin.

EMP are important not only because they relate to the quark orbital momentum contribution to the proton spin. They should test our understanding of QCD generally, and of OFPD's in particular. COMPASS may lead in the study of these new processes, that are only now calculable in QCD.

In principle, Deeply Virtual Compton Scattering represents the most elementary process to access the OFPD's. This reaction has been considered for COMPASS 6 . It was, however, realized that this channel may suffer from potentially large backgrounds. Detailed understanding of (exclusive) meson production is therefore a precondition for studying DVCS. In turn, DVCS may represent an important complement for the Exclusive Meson Production study, which we discuss below.

With the high muon beam energy and high luminosity in COMPASS experiment, large $Q^{2}$ values can be reached with reasonable rates. For $\rho_{0}$ for 
example, a $Q^{2}$ range between 2 and $20(80) \mathrm{GeV}^{2}$ is accessible for incident 100 $\mathrm{GeV} / \mathrm{c}(200 \mathrm{GeV} / \mathrm{c})$ muon beam. Thus, this COMPASS program extends the approved activity of the CLAS collaboration at JLAB OFPD in a limited $Q^{2}$ range up to about $4 \mathrm{GeV}^{2}$ We distinguish between EMP channels in which the final state meson $\left(\rho^{0}, \pi^{+}\right.$Q,$\phi$, etc. $)$ can be detected via magnetic spectrometers, and those which require electromagnetic calorimetry. The charged cases can be detected with higher resolution and more easily without EM calorimetry.

As another important aspect of this study, we also consider EMP of a "dipion" (both resonant and non-resonant), such as $\pi^{+} \pi^{-}, \bar{K} K, \pi K$, as studied recently by Polyakov 10 . These works demonstrated that the shape (not absolute cross sections!) of these "di-pion" mass distributions carry important information about quark distribution in the pion (kaon), and also about the structure of resonances $\left(\rho, f_{2}, K^{*}\right.$, etc.).

\section{The Proposed Experiment}

\subsection{Experimental methodology and trigger}

We base our considerations on the scattered muon trigger that is forseen in the COMPASS experiment to study spin dependent DIS. The trigger fires if the muon is scattered at large angle $\theta_{\mu}$. In our simulations we assumed a good angular acceptance for the range $10(5)<\theta_{\mu}<50 \mathrm{mrad}$ for $100(200) \mathrm{GeV}$ beam energy. The trigger is based on the $\mathrm{X}-\mathrm{Y}$ correlations between hodoscope elements at about 31 and 50 meters downstream of the target.

Average recoil proton energies for EMP are quite low $(\sim 30-40 \mathrm{MeV})$. Thus, the recoil proton kinematics for COMPASS do not allow installing an efficient proton recoil detector to isolate "elastic" reactions. In order to be able to make optimal use of the experimental setup employed by the main muon program, or even to run initially in parallel with the main program, we will evaluate the inelasticity $I \propto\left(M_{x}^{2}-M_{p}^{2}\right)$ using measured momenta of the incident and final muons and produced meson 11 . The resolution will, however, not be sufficient to cleanly resolve events with a recoil proton or a recoil $\Delta^{+}$or $\mathrm{N}^{*}$ in the final state. However, the OFPD's for these processes are related 12 . Thus, a comparison of the data should be made to model calculations which combine both, proton, $\Delta^{+}$and $\mathrm{N}^{*}$ production.

The main goal of the proposed program is to obtain high statistics EMP data on a pure unpolarized proton target during dedicated data runs. This would also help to avoid "dilution" by the presence of different target nuclei in the polarized proton target. Also, with the liquid hydrogen target, the detection efficiency of produced $\pi^{0}$ decay $\gamma^{\prime}$ 's would increase due to the bigger 
total radiation length of the LH. However, in order to explore the feasibility of this program already during the initial data taking period of COMPASS, we assumed the standard polarized target for our present simulations and count rate estimates.

\subsection{Cross section for exclusive meson production (EMP)}

We estimate the dependence of the cross section for the reaction $\mu p \rightarrow \mu p \pi^{0}$ on the kinematic variables $Q^{2}, \mathrm{x}_{B}$, and $\mathrm{t}$, using models for off-forward parton distributions. Following 3 , we constructed the relevant off-forward parton distributions starting from polarized valence quark distributions.

The exact QCD calculation involves time-consuming numerical integration, which is inconvenient for Monte Carlo purposes. However, neglecting QCD logarithmic evolution of off-forward parton distributions, one can obtain a simple parametrization based on a fit to the results of the exact formulae:

$$
\frac{d \sigma}{d t}\left(\gamma_{L} p \rightarrow \pi^{0} p\right)=\left.\frac{d \sigma}{d t}\left(x, Q^{2}\right)\right|_{t=\mathrm{t}_{\min }} \times e^{B\left(t-t_{\min }\right)},
$$

where:

$$
\left.\frac{d \sigma}{d t}\left(x, Q^{2}\right)\right|_{t=\mathrm{t}_{\min }}=\frac{\alpha_{S}^{2}\left(Q^{2}\right) \cdot \mathrm{PF}\left(x, Q^{2}\right) \cdot \mathrm{UF}(x)}{Q^{2}\left(Q^{2}+M^{2}\right)^{2}} .
$$

Here, $\alpha_{S}\left(Q^{2}\right)$ is the one-loop QCD coupling constant, which - as usual for LO calculations - was evaluated with $\Lambda_{Q C D}=0.2 \mathrm{GeV}$ and with $N F=4$ for the number of active flavors. In this equation, $\operatorname{PF}\left(x, Q^{2}\right)$ is given by:

$$
\operatorname{PF}\left(x, Q^{2}\right)=\frac{Q^{4}(1-x)}{\left(-Q^{2}-0.881721 x+Q^{2} x\right)^{2}} .
$$

$\mathrm{UF}(x)$ is the part of the cross-section which is independent of $Q^{2}$. It would acquire a logarithmic $Q^{2}$ dependence when QCD evolution effects are included. The shape of $\operatorname{UF}(x)$ depends on the model of the off-forward parton distributions used in the actual calculation.

Figure 2 shows the differential cross section for $\pi^{0}$ production at $\mathrm{t}=\mathrm{t}_{\min }$, $Q^{2}=4 \mathrm{GeV}^{2}$, as a function of Bjorken $\mathrm{x}_{B}$, in units of $n b / \mathrm{GeV}^{2}$, for the three different valence quark parametrizations. The solid line refers to the Gehrmann-Stirling polarized valence parametrization 15, the short-dashed line is based on the Carlitz-Kaur model 13, 16,17 and the long-dashed line results from the Bartelski-Tatur polarized valence distribution 18. We do not include twist-2 contributions to the OFPD, which according to recent calculations 2021 may change the absolute cross sections for EMP. 


\subsection{Monte Carlo results}

The events of exclusive $\pi^{0}$ production, $\mu+p \rightarrow \mu+p+\pi^{0}$ with the subsequent decay $\pi^{0} \rightarrow \gamma \gamma$, were generated with rates proportional to the estimates of differential cross sections from Ref. 18. The directly measured cross section could be expressed in terms of the virtual photoproduction cross section:

$$
\frac{\mathrm{d} \sigma_{\mu N \rightarrow \mu N \pi^{0}}\left(\nu, Q^{2}, t\right)}{\mathrm{d} \nu \mathrm{d} Q^{2} \mathrm{~d} t}=\Gamma\left(Q^{2}, \nu\right) \frac{\mathrm{d} \sigma_{\gamma^{*} N \rightarrow N \pi^{0}}\left(x=Q^{2} /\left(2 M_{p} \nu\right), Q^{2}, t\right)}{\mathrm{d} t},
$$

where $\Gamma$ is the flux of virtual photons:

$$
\Gamma=\frac{\alpha\left(\nu-\frac{Q^{2}}{2 M_{p}}\right)}{2 \pi Q^{2} E_{\mu}^{2}(1-\epsilon)} .
$$

Here, $\alpha$ is the fine structure constant, and $\epsilon$ is the virtual photon polarization.

The cross sections were estimated for proton and carbon targets. The latter one is more representative for the complex material of the COMPASS polarized target. For the proton target, $\mathrm{d} \sigma_{\gamma^{*} N \rightarrow N \pi^{0}}\left(x, Q^{2}, t\right) / \mathrm{d} t$ is given by the Eqs. (2) (4) and $B=4.5 \mathrm{GeV}^{-2}$. For $x=0.1, Q^{2}=4 \mathrm{GeV}^{2}$ and $t=t_{m i} \frac{d \sigma}{d t}$ is equal to $0.88 \mathrm{nb} / \mathrm{GeV}^{2}$. For similar conditions, Vanderhaeghen et al. 13 give about 4 times larger cross section. We will therefore give counting rate estimates in this report with an uncertainty, which reflects the difference between these two theoretical estimates. The present theoretical uncertainties are mainly due to different assumptions on the shapes/magnitudes of the valence quark spin distributions. For the carbon target, the cross section was assumed to be the product of the proton cross section and a function:

$$
f(t)=8.12 e^{-47.7\left(t-t_{\min }\right)}+0.3 .
$$

This function was obtained from the parametrization of the NMC data for exclusive $\rho^{0}$ production on a carbon target 19 .

In our simulations, we considered the kinematic range of $1 \mathrm{GeV}^{2}<\mathrm{Q}^{2}<$ $\mathrm{Q}_{\max }^{2} \mathrm{GeV}^{2}$, and $10(15) \mathrm{GeV}<\nu<85(180) \mathrm{GeV}$. Here, $Q_{\max }^{2}=4 E_{\mu}\left(E_{\mu}-\nu\right)$. $\sin ^{2}\left(\theta_{\mu}^{\max } / 2\right), \theta_{\mu}^{\max }=50 \mathrm{mrad}$, and $E_{\mu}$ is the incident muon energy. The values outside (inside) the brackets correspond to the beam energy of 100 (200) GeV. The same convention will be used hereafter. The upper cut on $\nu$ was chosen to eliminate the kinematic region where the amount of radiative events is large, whereas the lower one to eliminate events with poor kinematic resolution. The value of $Q_{\max }^{2}$ is about $20(90) \mathrm{GeV}^{2}$. The cross section integrated within this kinematic regime 


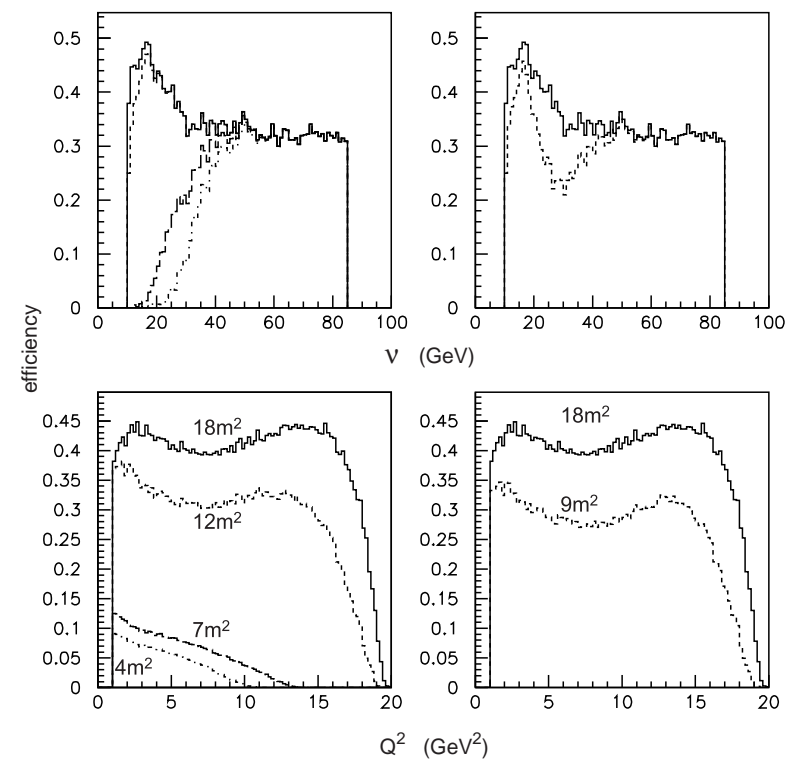

Figure 3: The total acceptance to observe exclusive $\pi^{0}$ events as a function of $\nu$ (top) and $Q^{2}$ (bottom) at a beam energy of $100 \mathrm{GeV}$. The different histograms correspond to different geometrical acceptances of the electro-magnetic calorimeter

The total acceptance $\epsilon_{\pi^{0}}$ to observe an exclusive $\pi^{0}$ event, taking into account the geometric acceptance, the absorption of the decay photons before entering the calorimeter, and the reconstruction of $\pi^{0}$ from the calorimetric measurements, is shown in the top parts of Fig. 3 as function of $\nu$ by the solid histograms. The main effect on $\epsilon_{\pi^{0}}$ is due to a poor reconstruction of low energy $\pi^{0}$ mesons from the calorimetric measurements, at small $\nu$, and due to the absorption of photons in the polarized target, most significantly at large $\nu$. The average total efficiency $\epsilon_{\pi^{0}}$ is 0.43 for both incident beam energies. For the kinematic range $\nu>15 \mathrm{GeV}, Q^{2}>1 \mathrm{GeV}^{2}, 0.02<x<0.2$, the efficiency is quite large, varying between 0.3 and 0.5 .

Taking the above values of cross sections, and the expected luminosity for the COMPASS experiment 0 of $43 \mathrm{pb}^{-1} \mathrm{day}^{-1}$, we evaluate the number of deep inelastic exclusive $\pi^{0}$ events per day, $N_{\mu \pi^{0} N}^{t o t} / \operatorname{day}\left(Q^{2}>1 \mathrm{GeV}^{2}\right)$, to be about 248-992 (53-212) for 100 (200) GeV. After taking into account effects of acceptance, secondary interactions, and the efficiency of $\pi^{0}$ reconstruction, the number of events, $N_{\mu \pi^{0} N}^{c u t s} / \operatorname{day}\left(Q^{2}>1 \mathrm{GeV}^{2}\right)$, is about 107-428 (23-92). The expected numbers of exclusive $\pi^{0}$ events for a running period of 150 days 
(corresponding to a 1 year period) assuming an overall (SPS and COMPASS) efficiency of $25 \%$ are $4013-16050$ (855-3420). For $Q^{2}>4 G e V^{2}$, we expect only 130 (42) counts. Adopting the Carlitz-Kauer valence quark parametrization the last numbers will increase by about a factor of 4 . Thus, in 1 year of COMPASS operation we will collect at most $500 \pi^{0}$ events with $Q^{2}>4 G e V^{2}$.

Up to this point we assumed full size electro-magnetic calorimeters (total of $18 \mathrm{~m}^{2}$ ) at their default positions. The different curves in Fig. 3 show the result if only limited coverage is assumed. While a reasonable efficiency may still be obtained with about half the calorimeter size $\left(9 \mathrm{~m}^{2}\right)$, a further cut (e.g. down to $7 \mathrm{~m}^{2}$ ) significantly reduces the $\pi^{0}$ efficiency. Furthermore, the count rates presented above were based on a polarized target. Assuming that the overall length of a target is limited to typically $2 \mathrm{~m}$, a dedicated LH target would lead to a reduced luminosity (about a factor of 4) unless the beam intensity can be correspondingly increased.

The detailed discussion of the possibility for COMPASS experiment to measure another exclusive vector meson production channel, $\mu+N \rightarrow \mu+$ $N+\rho^{0}$, is presented in Ref. 11 . Here we only briefly summarize the results of the Monte Carlo calculations for this reaction. The kinematic range is somewhat narrower than for the previous channel: $2 \mathrm{GeV}^{2}<Q^{2}<Q_{\max }^{2}$ and $20(40)<\nu<90(180) \mathrm{GeV}$. The estimated value of the measured cross section $\sigma_{\rho^{0}}^{\text {th }}$, is equal to $0.46 \mathrm{nb}(0.5 \mathrm{nb})$ for $100 \mathrm{GeV}(200 \mathrm{GeV})$. These values are obtained using a parametrization of the cross section measured in the NMC experiment 19. For the given kinematic range, the average total acceptance $\epsilon_{\rho^{0}}$ is 0.73 (0.68). Taking into account the expected luminosity for the COMPASS experiment, and the effects of acceptance and secondary interactions, the number of events with the invariant mass of two pions in the central part of $\rho^{0}$ peak, $0.62<m_{\pi \pi}<0.92 \mathrm{GeV}, N_{\mu \rho^{0} N}^{\text {cuts }} / \operatorname{day}\left(Q^{2}>2 \mathrm{GeV}^{2}\right)$, is about $10^{4}$ for both beam momenta. For a running period of 150 days assuming again an overall (SPS and COMPASS) efficiency of $25 \%$ this will result in about $3.7 \cdot 10^{5}$ events. For $Q^{2}>4 G e V^{2}$ we expect $75 \mathrm{k}(107 \mathrm{k})$ events.

\section{Conclusions}

We explored the feasibility to study exclusive meson production in hard muonproton scattering $\mu p \rightarrow \mu p M$ at the COMPASS experiment. For moderate $Q^{2}>1 \mathrm{GeV}^{2}$, a significant counting rate for exclusive $\pi^{0}$ production can be reached. For the experimental conditions assumed in our calculations, still up to 500 events beyond a $Q^{2}$ of $4 \mathrm{GeV}^{2}$ can be collected during 1 year of operation.

On the other hand, COMPASS may provide already during the first main 
$\mu$ runs, high statistics data on exclusive $\rho^{0}$ production even at $Q^{2}$ beyond values presently accessible at JLAB. Furthermore, data taken with the polarized target may also allow a first view at polarization phenomena.

\section{Acknowledgements}

This work was partially supported by the Polish State Committee for Scientific Research (KBN), by the U.S.-Israel Binational Science Foundation (BSF) and the Israel Science Foundation founded by the Israel Academy of Sciences and Humanities.

\section{References}

[1] A. Bravar, D. von Harrach, and A. Kotzinian, Phys. Lett. B421, 349 (1998).

[2] X. Ji, Phys. Rev. Lett.78, 610 (1997); Phys. Rev. D55, 7114 (1997).

[3] A.V. Radyushkin, Phys. Lett. B 380, 417 (1996); Phys. Lett. B385, 333 (1996); Phys. Rev. D56, 5524 (1997); hep-ph/9805342.

[4] I. V. Musatov and A. V. Radyushkin, Phys. Rev. D56, 2713 (1997).

[5] J. C. Collins, L. Frankfurt, and M. Strikman, Phys. Rev. D56, 2982 (1997).

[6] N. d'Hose, COMPASS collaboration meeting presentation (1998).

[7] COMPASS collaboration, CERN/SPSLC 96-14, SPSC/P 297.

[8] CLAS collaboration, JLab exp. E98-107/E99-105, spokespersons M. Guidal, C. Marchand and E.Smith (1998).

[9] L. Mankiewicz, G. Piller, A. Radyushkin, hep-ph/9812467.

[10] M.V. Polyakov, hep-ph/9809483, hep-ph/9901315, hep-ph/9906261.

[11] A. Sandacz, Compass Note 1998-5.

[12] L. Frankfurt, M.V. Polyakov and M. Strikman, hep-ph/9808449.

[13] M. Vanderhaeghen, P.A.M. Guichon, M. Guidal, Phys.Rev.Lett. 80 (1998) 5064; P. A. M. Guichon, M. Vanderhaeghen, Prog. Part. Nucl. Phys. 41125 (1998); hep-ph/9806305.

[14] L. Mankiewicz, G. Piller, and T. Weigl, Eur. Phys. J. C5 119 (1998).

[15] T. Gehrmann and W. J. Stirling, Phys. Rev. D53, 6100 (1996).

[16] R. Carlitz and J. Kauer, Phys. Rev. Lett.38, 673 (1977).

[17] M. Goshtasbpour and G.P. Ramsey, Phys. Rev. D55, 1244 (1997).

[18] M. Kurzela, J. Bartelski, S. Tatur, hep-ph/9807355.

[19] NMC collaboration, M. Arneodo et al., Nucl. Phys. B429, 503 (1994).

[20] V. Yu. Petrov et al., Phys. Rev. D57, 4325 (1998).

[21] M.V. Polyakov and C. Weiss, hep-ph/9902451. 